\title{
Processamento da informação em gestores de alto desempenho
}

\author{
S.C. Garcia-Santos, L.S. Almeida, B.S.G. Werlang, A.L.M. Veloso
}

O propósito deste estudo foi avaliar o processamento da informação de 43 gestores empresariais de desempenho superior. No seu enquadramento teórico recorremos a três modelos: a Teoria dos Papéis Gerenciais de Henry Mintzberg, a Teoria do Processamento da Informação e o Modelo de Processo de Resposta ao Rorschach de John Exner. A avaliação dos sujeitos recorreu ao método do Rorschach. Os resultados deste estudo mostraram que esses gerentes são capazes de recolher dados, avaliá-los e estabelecer rankings de maneira adequada. Ao mesmo tempo, verifica-se que são capazes de ser objetivos e precisos na avaliação dos problemas. Este estilo de processamento de informação possibilita uma interpretação do mundo a partir de um modo de processamento ou de um estilo cognitivo, próprio e característico.

Palavras-chave: excelência, processamento da informação, estilo cognitivo, Rorschach, gestores

\section{Information processing among high-performance managers}

The purpose of this study was to evaluate the information processing of 43 business managers with a professional superior performance. The theoretical framework considers three models: the Theory of Managerial Roles of Henry Mintzberg, the Theory of Information Processing, and Process Model Response to Rorschach by John Exner. The participants have been evaluated by Rorschach method. The results show that these managers are able to collect data, evaluate them and establish rankings properly. At same time they are capable of being objective and accurate in the problems assessment. This information processing style permits an interpretation of the world around on basis of a very personal and characteristic processing way or cognitive style.

Keywords: excellence, information processing, cognitive style, Rorschach, managers

Seille Cristine Garcia-Santos e Blanca Susana Guevara Werlang. Pontifícia Universidade Católica do Rio Grande do Sul, Brasil.

Leandro S. Almeida e Ana Luísa Marques Veloso. Universidade do Minho, Portugal.

Endereço para correspondência: Leandro S. Almeida, Universidade do Minho, Instituto de Educação e Psicologia, Campus de Gualtar, 4710-057 Braga - Portugal

E-mail: leandro@iep.uminho.pt 
Em 1994, Henry Mintzberg, investigador canadiano da área da gestão, apresentou uma actualização da sua Teoria dos Papéis da Gestão, lançada em 1975. Neste trabalho, o autor explicou que os papéis da gestão, por si agrupados nas dimensões interpessoais, informacionais e decisionais, são desempenhados a três níveis mediando as relações internas e externas à organização. O primeiro nível refere-se à gestão de informações, o segundo das pessoas e o terceiro trata das acções propriamente ditas. Segundo Mintzberg (1994), nenhum dos papéis de gestão, mesmo que exercidos adequadamente, dispensa as tarefas compreendidas nos demais pois todos eles constituem um todo integrado ou uma gestalt.

Para aplicar os pressupostos teóricos de Mintzberg $(1975,1994)$ nos dias de hoje é necessário lançar um olhar aos fenómenos da globalização, especialmente na alteração provocada pela utilização massiva das novas tecnologias da informação na vida das pessoas e nas relações humanas. Neste campo, o tempo foi alterado parecendo não seguir o mesmo ritmo do ponteiro do relógio, isto é, a marcação do tempo recebeu outro tratamento nas organizações estando agora associado às entradas e saídas de informações em tempo real. Isso parece fazer do gestor um importante catalisador de informações, pois é múltipla a informação interna e externa a que tem acesso, sendo necessário filtrar, processar e dar sequência de forma a assegurar a produção, a monitorização das estratégias organizacionais e a manutenção parcimoniosa das relações externas à organização. Ao mesmo tempo, a globalização extinguiu distâncias, estreitou mercados, redireccionou as grandes empresas e o seu funcionamento. Com tudo isto, as organizações necessitam de pessoas ágeis, adaptáveis e perspicazes em todos os níveis hierárquicos, mas especialmente ao nível da gestão (Silvius, 2009). Em síntese, o contexto organizacional é bastante diferente de há 15 anos atrás: a era da tecnologia da informação onde o processo de trabalho é globalizado, acontece em tempo real e é determinado pela alta competitividade que isso ocasiona. Mais ainda, a evolução no mundo de trabalho segue em percurso ininterrupto e requer elevada capacidade de gestão.

Depreende-se a relevância do tratamento de informação por parte do gestor (Mintzberg, 1994) e o seu status decorre de uma actividade permanente a processar, filtrar e difundir informações para que a organização evolua. Em estudos recentes que procuraram replicar a investigação de Mintzberg, como por exemplo o de Tengblad (2006), concluiu-se também pela relevância da componente de gestão da informação na função do gestor, mesmo quando importantes mudanças ocorreram nomeadamente ao nível da concepção actual de liderança, e pela menor importância da vertente administrativa da função de gestão. Realça-se pois, a ênfase e a importância inegável do papel informacional da função de gestor.

Face ao fluxo actual de informações a que tem que dar atenção, concordamos com Mintzberg (Mintzberg, 1994, 2006; Mintzberg \& Gosling, 2003) 
quando refere que o desempenho dos papéis de gestão é determinado por disposições mentais, ou seja, um conjunto de capacidades e de conhecimentos que forjam a interpretação do mundo ao seu redor, a partir de um modo de processamento ou de um estilo cognitivo associado à sua personalidade $\mathrm{e}$ motivações pessoais. Outros autores, como por exemplo Muller e Turner (2009) incluíram este aspecto da função de gestão, ainda que com outra designação (Intellectual competence: Critical analysis and judgment), enfatizando a importância do processamento de informação por gestores de sucesso (p. 11). A identificação do perfil de gestores bem sucedidos com o objectivo de reconhecer as dimensões psicológicas que contribuem para o seu sucesso, resulta da constatação de que se estes tiverem as características adequadas às exigências da sua função, o seu sucesso será garantido e a sua permanência em lugares de topo, longa. Conclui-se que, se a função de gestão inclui o processamento de informações, então o desempenho está dependente do estilo pessoal do gestor no manuseamento das informações.

A partir desta proposição, associada ao cenário empresarial mundial contemporâneo, pretende-se neste artigo avaliar e compreender as condições do processamento das informações em gestores de elevado desempenho que trabalham em grandes empresas brasileiras (da cidade de Porto Alegre, Rio Grande do Sul). Mais concretamente procura-se observar o que revelam esses gestores quando confrontados com situações-problema inusitados que devem ser resolvidos de forma veloz e eficiente para garantir o bem da própria empresa. Para responder ao objectivo deste estudo recorre-se a um método projectivo de avaliação da personalidade. Este método possibilita a investigação do processamento da informação por meio das respostas a um grupo de manchas de tinta fortuitas (de Hermann Rorschach) que passam a ser, da forma como são apresentadas, um problema a ser resolvido e desencadeiam no indivíduo uma série de processos cognitivos, até que tome a decisão a respeito do que vê e emita uma resposta (cf. Exner, 1989).

$\mathrm{Na}$ interpretação dos dados empíricos obtidos com o Método de Rorschach pretende-se aproveitar as contribuições de outras duas abordagens teóricas: a Teoria dos Papéis de Gestão, especificamente o papel informacional do gestor como comunicador interno e externo à organização e por isso centralizador da solução de problemas (Minzberg, 1975, 1994) e a Teoria do Processamento da Informação e a sua forma de entender a sequência dos processos cognitivos envolvidos na resolução de problemas (Hunt, 1980; Simon, 1981; Sternberg, 2000). O conhecimento gerado a partir do entrelaçamento destas teorias almeja contribuir para a preparação, a formação e o desenvolvimento de indivíduos cada vez mais capacitados e talentosos para fazer frente às exigências empresariais actuais. 
A Teoria dos Papéis Informacionais de Mintzberg

Quando Mintzberg (1975) sintetizou os resultados de suas pesquisas e publicou The manager's job: folklore and fact explicitou, naquele momento, um enfoque diferenciado acerca da função de gestão. $\mathrm{O}$ autor redefiniu paradigmas clássicos, sugerindo que o trabalho do gestor pode ser descrito em termos de vários papéis. Especificamente, menciona dez papéis divididos nas dimensões interpessoal, informacional e decisional. Assim, decorrente da autoridade formal de que são investidos os gestores, deriva uma posição organizacional que o leva a várias relações interpessoais de acesso à informação, o que o capacita a tomar decisões e a formular estratégias para o desempenho de sua função. Os papéis interpessoais de que fala Mintzberg (1975) referem-se a: protocolo (figurehead) que compreende todas as tarefas relativas às funções simbólicas e cerimoniais que demarquem uma posição legal ou social; líderança (leader), responsável pela motivação e direcção dos colaboradores; e ligação (liaison) eficiente com o ambiente externo (fornecedores, parceiros, concorrentes e pares) e ambiente interno (superiores e subordinados) à organização. Já os papéis decisionais, segundo o autor, reflectem a responsabilidade que os gestores têm para com a implementação das escolhas que fazem. São eles: empreendedorismo/inovação (entrepreneur), promovendo as mudanças adaptativas ao funcionamento relativo ao meio externo; resolução de problemas (disturbance handler), no sentido de implementar acções correctivas e preventivas de conflitos; afectação de recursos (resource allocator), definindo o que e quando fazer, tanto com os recursos materiais quanto com humanos; e, negociação (negotiator) em todos os níveis da empresa.

Alterando a ordem dos papéis proposta por Mintzberg (1975), e de acordo com os objectivos deste estudo, descrevem-se, por último, os papéis informacionais que são os de monitorização (monitor) da busca activa de informação junto a subordinados e da rede de contactos para ampla recolha de informações; difusão (disseminator) das informações, já elaboradas e filtradas, directamente aos subordinados e pessoas do seu grupo de trabalho; e, porta-voz (spokesman), transmissor das informações, elaboradas e filtradas, a pessoas externas ao seu grupo mais próximo de trabalho. Em função do papel informacional, o gestor para Mintzberg (1975) transforma-se numa espécie de 'centro nervoso' da organização, e isso ocorre devido ao delicado equilíbrio que é imposto à função. $\mathrm{O}$ gestor recebe uma grande quantidade de informações externas que podem influenciar as suas decisões, assim como, também recebe informações internas de superiores, pares e subordinados. Todas elas necessitam ser processadas, a partir do seu background cognitivo, sem perder o foco na organização, nos interesses colectivos, e na actualidade, e considerando um complexo espectro de possibilidades, em alta velocidade, e acertadamente.

No desenvolvimento da sua teoria, Mintzberg (1994) refere que os papéis 
são desempenhados a três níveis que compõem o Modelo Integral do Trabalho de Gestão. Em outras palavras, esclarece o autor, o gestor está no centro e é solicitado a desempenhar os papéis que são executados sucessivamente desde a gestão pelas informações, passando pela gestão através das pessoas e finalizando com a gestão através de acções.

$\mathrm{Na}$ gestão pelas informações, Mintzberg (1994) reforça a supremacia da capacidade de gestão da comunicação e controlo. A comunicação implica basicamente a recolha e disseminação das informações. A recolha é feita em todos os contactos que são estabelecidos, dedicando os gestores grande esforço e atenção às informações de duplo-sentido ou ambíguas, que advém da informalidade. A resolução de problemas advindos de informações formais (relatórios, boletins, planos, etc.) torna-se por contraposição mais simples. Ao concluir o processamento das informações, o gestor dissemina-as dentro e para fora da organização, assumindo, nesta circunstância, o papel de porta-voz, sem perder o foco nos objectivos estratégicos da empresa. Quando fez esta proposta, Mintzberg $(1975,1994)$ enfatizou o carácter do papel informacional no trabalho de gestão, afirmando que esse papel é dependente das preferências e do 'modelo mental' do gestor. Contudo, não esclareceu como, na sua teoria, ocorre o processo de tratamento das informações, propriamente dito.

\section{A Teoria de Processamento da Infor-}

\section{mação}

Em meados do século passado, em reacção à abordagem comportamentalista vigente, nasceu uma nova abordagem da Psicologia, o cognitivismo. Os fenómenos mentais e os processos neles envolvidos ganharam relevância e valor. As investigações passaram a focar a identificação dos processos cognitivos, ou seja, como as pessoas apreendem a informação e como agem sobre a informação, centrando-se especificamente nos processos de atenção, percepção e memória (Almeida, 1994; Huitt, 2003; Resnick, 1976; Sternberg, 1981). Assim, segundo Almeida (1994), mais que avaliar traços (aptidão mental, quociente de inteligência), os estudos voltaram-se para os processos de atenção e codificação da informação, o seu tratamento e a resposta ou resolução.

Foi a partir de então, levando em conta o método envolvido na recolha de informação, a forma de elaboração mental e as respostas advindas do processo, que surgiu a teoria do processamento da informação concomitante ao advento dos computadores e ao final da Segunda Guerra Mundial. Nesta nova abordagem considerou-se, segundo Simon (1981), que os processos mentais eram comparáveis aos softwares executados nos computadores. Foi deste modo, explica Simon (1981), que surgiram abordagens preocupadas com os níveis mais elementares do processamento da informação (envolvendo acesso à memória, classificação das listas comparativas na memória, comparação simbólica simples - ver Chase 
\& Simon, 1973; Reitman, 1976; Simon \& Gilmartin, 1973) e, outras, com os processos mentais superiores que envolvem a resolução de problemas e tomada de decisão (Newell, Shaw, \& Simon, 1958; Simon \& Newell, 1972).

Para se entender o processo de resolução de problemas, ênfase deste estudo, é preciso retomar o modelo de tratamento da informação. Propõe-se que alguns processos cognitivos podem ser assumidos como gerais a todas as tarefas, inclusive as de resolução de problemas (Almeida, 1994; Hunt, 1980). Entre eles, estão os processos de atenção e percepção, com os quais o indivíduo capta, apreende e codifica (input), dando significado e organização às informações (externas ou internas) que lhe chegam ao 'executivo central' (também chamado de campo de consciência, de atenção ou memória de trabalho); em seguida, intervêm os processos de categorização e relacionamento, integrando a informação disponível na memória de longo-prazo possibilitando lidar com a complexidade do problema (treatment). Por fim, ocorrem os processos de avaliação e justificação associados à produção ou à escolha de uma determinada resposta para o problema em processo de solução (output).

Ao longo das fases da resolução do problema, a capacidade resolutiva do sujeito - avaliada em termos de qualidade, exactidão e velocidade - irá depender, em termos cognitivos, do grau de destreza nas operações mentais envolvidas; e também do grau de conhecimento da informação em que o problema se expressa ou nas noções requeridas para a sua resolução. Sobre as operações necessárias para a resolução do problema, Sternberg (2003) inclui as meta-componentes associadas ao planeamento da execução, ao mapeamento de estratégias, à monitorização da realização ou à avaliação da qualidade das respostas. Da mesma forma, sobre o conhecimento necessário para a resolução do problema, os autores diferenciam a maior facilidade de realização quando o sujeito domina os conteúdos ou quando já os possui automatizados. Por outro lado, a dificuldade é aumentada quando a informação é pautada pela novidade. Aliás, comparando-se sujeitos especialistas (experts) e inexperientes (novices), os autores destacam o nível e a qualidade do conhecimento (informação) possuído para diferenciar o desempenho comparado destes dois grupos de indivíduos. O especialista (expert) não só possui mais informação na memória de longo prazo, como a tem melhor organizada e hierarquizada, permitindo-lhe acesso mais fácil. Uma vez que as unidades de processamento da informação disponíveis na memória de trabalho são limitadas, segundo Miller (1956), quanto mais organizada e combinada estiver a informação, melhor, pois deixa mais unidades disponíveis para o trabalho de relacionamento da informação.

Após vários anos de debates e evolução da teoria do processamento da informação, um modelo de ciclo de solução do problema foi proposto por Sternberg (2003); um dos exemplos ilustrativos utilizado pelo autor foi justamente o tratamento da informação a 
ser efectuado por um indivíduo em função de gestão. Propõe Sternberg (2003), que o gestor deve seguir criteriosamente cada etapa do ciclo para que consiga lidar com o problema e encontrar a melhor solução. Segundo o autor, o termo ciclo é utilizado porque, usualmente, a solução de um problema acaba por ser a base para um próximo problema e é composto por sete etapas: a) reconhecimento da existência do problema; b) definição da natureza do problema; c) alocação de recursos para solucionar o problema, d) representação mental do problema; e) formulação de estratégias para solucionar o problema; f) acompanhamento do problema enquanto a solução está em curso e g) avaliação da solução do problema após a sua implementação. Cada etapa do processamento da informação que se encadeia no ciclo de solução do problema é importante, porém, são especialmente significativas as etapas de reconhecimento e definição do problema, a ponto de, se o problema não for adequadamente percebido, não ser encontrada uma solução.

\section{O Modelo de Processo de Resposta de}

\section{Exner}

Em 1974, John E. Exner publicou nos Estados Unidos, The Rorschach: $A$ compreensive system, integrando cinco sistemas de codificação e interpretação existentes à época, naquele país. Uma das propostas do autor do novo sistema de tratamento dos dados do Rorschach foi a de questionar, em 1989, "onde está a projecção no Rorschach?” parecendo remeter para a formulação inicial do próprio Hermann Rorschach, no seu livro Psicodiagnóstico: métodos e resultados de uma experiência diagnóstica de percepção (Interpretação de formas fortuitas), em 1921. Naquele momento, o autor do método das manchas de tinta, enfatizava os elementos perceptivos que as manchas evocavam, para somente mais adiante falar em projecção. Depreende-se que Rorschach (1921/1974) colocou a percepção na base do método, como meio de acesso a alguns aspectos da personalidade. Seguindo o pensamento de Exner (1989), é possível entender o modelo cognitivo de processo de resposta, criado a partir das manchas do Rorschach.

Exner (1989) afirma que somente um pequeno número de respostas num protocolo do Rorschach pode envolver alguma projecção e, ainda assim, de difícil identificação. Alguns tipos de respostas podem ser mais indicativas de projecção, porém, mesmo estas devem ser consideradas com cautela, explica o autor. Entre as respostas que Exner (1989) descreve como sugestivas de projecção, estão as que envolvem movimentos passivos, agressivos, conteúdos mórbidos e de movimentos humanos ou animais em atitude de interacção cooperativa, por exemplo. Neste caso, a projecção é entendida como a atribuição de qualidades e necessidades próprias do indivíduo aos estímulos contidos nas manchas do Rorschach, sem que ele tome consciência e se aperceba disso (Werlang \& Cunha, 1993).

Sabe-se, porém, que nenhuma resposta ao Rorschach é acidental ou ao acaso. Cada uma é produto de um complexo processo de operações psico- 
lógicas que culminam na emissão de uma resposta (Acklin \& Wu-Holt, 1996; Exner, 1989; Gold, 1987). Partindo desta premissa, Exner (1974, 1978, 1989) descreve a resposta como um processo de elaboração, o que não deixa de ser semelhante, em alguns aspectos, aos modelos teóricos anteriores. Contudo, distancia-se no que se refere aos enfoques mais associativos e projectivos destacados por alguns (Rapaport, 1946; Schafer, 1954). No processo de resposta proposto por Exner (1989), parte-se do princípio que a mancha de tinta funciona como um estímulo visual, bastante ambíguo, que produz uma complexa operação cognitiva mediada pela forma particular de o indivíduo examinar a mancha, pela característica específica de cada mancha (por exemplo, a mancha do cartão I é mais compacta, enquanto a do cartão III é mais difusa), pela influência exercida pelo ambiente de exame (factor de grande relevância para o resultado; é necessário um ambiente facilitador e cooperativo) e, também, pela própria personalidade e estilo cognitivo da pessoa em avaliação.

A partir do momento em que um indivíduo recebe um cartão do Rorschach e ouve a questão "O que isto poderia ser?" várias fases do processo de resposta são desencadeadas, segundo Exner (1989), sendo a primeira composta pela codificação e classificação da mancha. Nesta fase, segundo o autor, o indivíduo perscruta a mancha muito rapidamente e codifica o que percebe; uma vez iniciada a codificação, informações de longo prazo armazenadas são imediatamente acedidas e o indivíduo começa a realizar comparações com a imagem que está sendo codificada. Essas comparações conduzem a classificações da mancha ou de partes dela, desencadeando o delineamento de possíveis respostas à indagação feita. É um processo muito rápido, que gera uma espécie de lista de respostas em potencial.

A segunda fase é a mais complexa e a que consome mais tempo de processamento, como explica Exner (1989), é o momento de ordenar um ranking de respostas em potencial e descartar aquelas que não são adequadas perante o julgamento efectuado. Significa dizer que o indivíduo, após a classificação das possíveis respostas, irá revê-las estabelecendo uma ordem ou ranking para, em seguida, rejeitar aquelas respostas em potencial que não cumprem os requisitos do processo de julgamento de mérito, utilizado na avaliação de cada resposta ordenada. A rejeição é baseada em parâmetros comparativos sendo que muitas respostas são rejeitadas, enfatiza o autor, em função da censura relacionada ao seu conteúdo (como por exemplo: respostas envolvendo alguma violência, sexualidade ou outros elementos que interfiram naquilo que seria 'socialmente aceite' para aquela situação de avaliação).

$\mathrm{Na}$ última fase do processo de resposta ocorre a selecção final e a articulação da resposta seleccionada. Para Exner (1989), esta fase é uma extensão da anterior, mais simples e de menor envolvimento cognitivo. $\mathrm{O}$ processo de censura das respostas é concluído, o indivíduo elege a resposta que será articulada e a emite. Entenda-se que 
este processo será repetido para cada resposta verbalizada, em cada cartão do Rorschach. A figura 1 apresenta esquematicamente as três fases do processo de resposta de Exner.

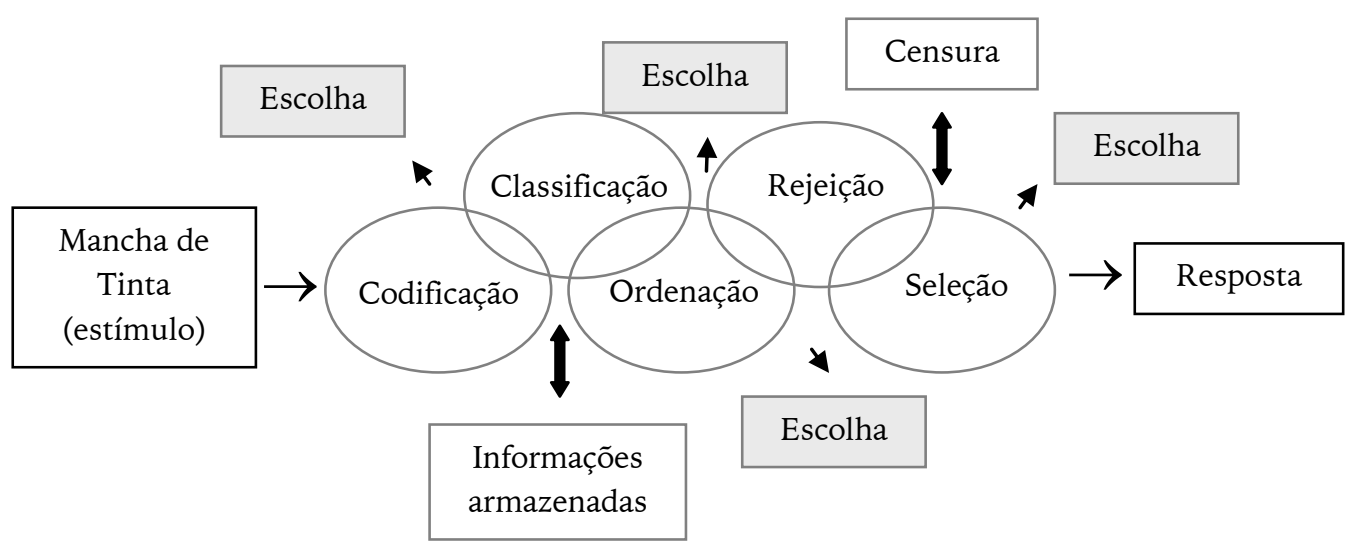

Figura 1. Ilustração do Modelo de Processo de Resposta proposto por J. Exner (1989)

As três fases não são distintas, elas se sobrepõem e ocorrem num processo contínuo, sendo que algumas operações são retomadas de modo mais refinado a cada fase e outras podem se repetir em momentos diferentes (Acklin \& WuHolt, 1996; Exner, 1989). Uma das operações cognitivas que está presente nas três fases é a tomada de decisão. $\mathrm{Na}$ primeira fase, o indivíduo elege se irá utilizar o todo da mancha ou apenas partes dela para codificar. De seguida, inicia a escolha das informações armazenadas de longo prazo que irá utilizar na classificação. A partir de então, escolhe a ordem do ranking para decidir quais das respostas em potencial irá descartar e quais irá enviar para a fase de selecção (que já é uma escolha), após ter passado pela avaliação e decidir, finalmente, respondendo à pergunta sobre o que pode ser aquela mancha que lhe foi apresentada.

Como se nota, a emissão de uma resposta no Rorschach é um trabalho complexo relacionado com a resolução de um problema proposto (Yazigi \& Gazire, 2002); envolve a activação de vários processos cognitivos que abarcam a atenção visual e a percepção, o reconhecimento do objecto, a memória associativa, a produção de linguagem e as funções executivas num esquema de funcionamento que requer refinada organização (Acklin \& Wu-Holt, 1996; Gold, 1987; Smith, Bistis, Zahka, \& Blais, 2007). Assim, parece possível tecer a intersecção das três abordagens, já que Exner (1989), partindo de um entendimento cognitivista do Rorschach, valoriza o processamento $\mathrm{da}$ informação nas respostas. As etapas em que se fundamentam as abordagens, sobrepõem-se e ajustam-se desde o momento de exposição ao estímulo até a finalização do processo com a emissão da resposta e a resolução do problema. Da mesma forma, ainda que numa perspectiva da gestão, Mintzberg (1994) ao afirmar que processar informações é uma tarefa central da função do gestor, ou seja, as informações (em 
forma de problemas, dados e situações) chegam, são tratadas e distribuídas pelo gestor em fluxo constante, precisam ser filtradas e adequadas ao objectivo a que se destinam, exigindo do indivíduo, nesta posição, elevada capacidade de processamento das informações.

\section{O Rorschach na avaliação do Proces-}

\section{samento da Informação}

Algumas variáveis avaliadas no Rorschach permitem revelar o processamento da informação de um indivíduo. Conforme Sendín (1999, p.71), essas variáveis permitem observar “[...] como o indivíduo incorpora as informações procedentes do exterior, como as identifica e traduz para outros códigos e como elabora conceitos a partir dela". Este tipo de operação psicológica, esclarece a autora, ocorre de modo muito complexo e tem um papel muito importante no comportamento que o indivíduo inicia deliberadamente. Além disso, a qualidade dos processos envolvidos nestas elaborações, representadas nas percepções em forma de respostas ao Rorschach, diz o quanto o indivíduo será mais ou menos capaz de se ajustar às exigências da sua realidade quotidiana. Em outros termos, observam-se na apreensão das condições de processamento da informação no Rorschach as seguintes variáveis, segundo Exner (1999) e Sendín (1999):

a) Lambda: afere a proporção de respostas de forma pura, expressa o quanto o indivíduo simplifica suas percepções ou o quanto as elabora e sofistica. As respostas de forma pura (F) são consideradas as mais simples e de menor dispêndio de energia que pode aparecer num protocolo do Rorschach. Estas respostas simples quando em número elevado podem representar $\mathrm{O}$ quanto o indivíduo recorre ao controle intelectual, evitando processar as emoções, mesmo que com isto perca a captação de elementos chaves da informação externa traduzindo um estilo super simplificador. Por outro lado, indivíduos com número muito baixo de respostas mais simples podem deixarse invadir facilmente pela estimulação emocional, ou seja, o processamento da informação sofre interferência de elementos emocionais carregados de informações acessórias, o que se traduz na vida real por uma diminuição de sua eficiência na solução de problemas. Esperase, segundo dados normativos para a população brasileira (Nascimento, 2007), que Lambda esteja em torno de $.98(D P=$ 1.11).

b) OBS e HVI: referem-se ao estilo obsessivo (OBS), com uma marcada tendência ao perfeccionismo, e ao hipervigilante (HVI) com acentuado estado de alerta. Nos indivíduos com OBS positivo, a recolha da informação ocorre de modo minucioso, detido em meticulosidades. Resulta num comportamento preocupado em não falhar, não errar e por isso, estes indivíduos 
esforçam-se para serem mais convencionais, diminuindo a sua capacidade de inovar criativamente, em função do que é correcto ou previamente acordado, ao transformar a informação processada em acção. Já os HVI positivos denotam um processamento muito cuidadoso e pertinente na recolha da maior quantidade possível de dados a respeito do problema em análise, com impacto significativo no ajustamento da sua produção ao meio.

c) Actividade organizativa (Zf); proporção das localizações (W : $\mathrm{D}$ : Dd); proporção glo$\mathrm{bal} /$ movimento humano (W : $\mathrm{M})$ : de maneira geral, a interacção destes aspectos diz o quanto o indivíduo se esforça na actividade do processamento da informação (índice de economia - W : D : Dd), como ocorre o processo de recolha e elaboração da informação e com que motivação é capaz de organizar um problema (índice de aspiração W : M). Cada vez que um indivíduo emite uma resposta, para o que necessita organizar e atribuir um sentido, criando uma relação significativa entre elementos fortuitos, põe em jogo elementos cognitivos, afectivos e emocionais, assinalando um esforço que pode, ou não, ser criativo. Todas as relações que estabelece para criar uma resposta, gastam alguma energia. Porém, haverá aquelas mais complexas, de maior grau de dificuldade na sua estruturação e que precisam ser valorizadas por isso. Sempre que o indivíduo organiza o campo de estímulos, dá à tarefa um nível de esforço maior do que ao dar uma resposta simples e pouco elaborada (uma D, por exemplo). Espera-se que Zf, se situe em torno de $40 \%$ das respostas (R), W próximo de $40 \%$ do protocolo, assim como D em $60 \%$ e Dd em torno de $5 \%$ de R. Já a proporção de W:M deve situarse em torno de $2: 1$.

d) Análise da qualidade evolutiva (DQ): a avaliação da DQ associada às outras variáveis já apresentadas, representa a forma pela qual o indivíduo trabalha cognitivamente a área de localização que escolheu. Relacionase directamente com o nível de desenvolvimento intelectual e a capacidade de realizar operações de análise e síntese e actuar proactivamente na resolução de problemas. Observam-se as modalidades de elaboração se: sintetizada $(+)$, ordinária $(0)$, vaga (v) ou sintetizada (v/+); assinala-se que a maior sofisticação no processamento da informação para análise e síntese ocorre nas respostas $(+)$.

e) Análise da eficiência do processamento $(\mathrm{Zd})$ : investiga sobre a recolha e codificação da informação, nomeadamente a precisão e o registo daquilo que é central no problema apresenta- 
do. É possível uma oscilação entre -3 e +3 pontos para $\mathrm{Zd}$, sendo que o intervalo entre os extremos é comum em pessoas com adequação na recolha e codificação das informações. Já os indivíduos com valores abaixo do índice inferior são descritos por um estilo subincorporador na maneira de examinar um problema, sendo pouco cuidadosos, o que pode ser manifesto em precipitações na tomada de decisão. Aqueles que se situam acima da margem superior, são referidos por um estilo hiper-incorporador que se reflecte em excesso de meticulosidade na análise da informação, o que apesar de diminuir o risco de erro, dificulta a discriminação daquilo que é essencial para a solução de um problema.

Sendín (1999) sugere também que se analisem as perseverações (PSV) e a sequência em que ocorrem as localizações. Em função dos objectivos que se pretendem alcançar neste estudo, serão utilizadas as variáveis destacadas nos itens de $a$ ) a $e$ ).

\section{MÉTODO}

\section{Amostra}

Os participantes deste estudo são gestores de nível estratégico, provenientes de sete empresas de grande dimensão (das áreas do comércio, comunicação, educação superior, financeira, indústria e saúde) de Porto Alegre, Rio Grande do Sul - Brasil, privadas e de capital nacional. A amostra, por conveniência, é composta por 43 gestores brasileiros, 25 do sexo masculino e 18 do sexo feminino. A idade do grupo situa-se entre 27 e 64 anos, sendo 31 casados, oito solteiros e quatro divorciados e têm em média 8.7 anos de tempo de experiência como gestor. Todos eles foram avaliados por suas empresas como de elevado desempenho nas suas actividades.

Para classificar com segurança a dimensão da empresa foram consultadas as seguintes instituições: Banco Nacional de Desenvolvimento Social (BNDES); Federação e Centro das Indústrias do Estado do Rio Grande do Sul (FIERGS); Instituto Brasileiro de Geografia e Estatística - Cidades Brasileiras (IBGE.Cidades@); Fundação de Economia e Estatística do Estado (FEE); Sindicato dos Estabelecimentos do Ensino Privado no Estado do Rio Grande do Sul (SINEPE/RS); Federação dos Hospitais do Rio Grande do Sul (FEHOSUL); Sindicato dos Hospitais e Clínicas de Porto Alegre (SINDIHOSPA); Federação das Associações Comerciais e de Serviços do Rio Grande do Sul (FEDERASUL) e Federação do Comércio de Bens e de Serviços do Estado do RS (FECOMÉRCIO).

Estes órgãos indicaram dois critérios para a classificação da dimensão da empresa:

a) são empresas de grande dimensão pelo BNDES (2005) aquelas que tenham obtido receita operacional bruta anual acima de $\mathrm{R} \$ 60$ milhões (sessenta milhões de reais) e,

b) pela FIERGS (2006) as empresas que tenham mais de 500 funcionários. 


\section{Instrumentos}

Foi utilizada uma ficha de dados sociodemográficos para o levantamento de dados pessoais dos gestores, e para avaliar o processamento da informação foi utilizado o Método de Rorschach. $\mathrm{Na}$ codificação e interpretação das respostas ao Rorschach foi utilizado o Sistema Compreensivo (Exner, 1999; Exner \& Sendín, 1999; Nascimento, 2007; Weiner, 2000). Para obter dados sobre o objecto de investigação foram avaliadas as seguintes variáveis do Rorschach: número de respostas (R); proporção de respostas de forma simples (Lambda); índices, OBS e HVI; frequência de nota $Z(Z f)$ e a diferença para Zestimada $(Z d)$; localizações globais, detalhe comum e incomum (W, D e $\mathrm{Dd}$ ); movimento humano (M); qualidade evolutiva sintetizada (DQ+).

\section{Procedimentos}

Após a aprovação do projecto pelo Comité de Ética da Pontifícia Universidade Católica do Rio Grande do Sul os gestores indicados pelos departamentos de Recursos Humanos das empresas, foram convidados a participar. Com a aceitação do convite foram agendados os horários. Os instrumentos foram administrados de forma individual pela primeira autora. Foi realizada a codificação dos doze primeiros protocolos pela mesma investigadora e por um juiz experiente no método, para cálculo do índice de concordância por meio do teste estatístico Kappa (o valor obtido foi de .85 para os determinantes, .94 para os conteúdos e .98 para as localizações). Conforme Exner e Sendín (1997), se a concordância entre o psi- cólogo que aplica o Rorschach e o juiz for boa (neste caso a concordância foi altamente satisfatória, já que zero representa uma concordância menor, ou mesmo ausência, e um uma concordância perfeita,) em pelo menos $20 \%$ dos casos da amostra, é possível que o investigador faça a codificação dos demais casos sem a necessidade da avaliação de todos os casos por juízes. Foi este o procedimento seguido neste estudo, sendo que para dirimir as divergências que ocorreram nos doze casos, os juízes retomaram cada uma das respostas com discordância e chegaram a uma codificação consensual, adoptada para a interpretação assumida. Os dados do Rorschach (codificados em: localizações, determinantes, conteúdos e códigos especiais) foram lançados no software Rorschach Interpretation Assistance Program, v 5.0, para realização da estatística descritiva.

\section{RESULTADOS}

O método de Rorschach possibilitou o levantamento dos aspectos relativos ao processamento da informação. Foram levadas em conta as informações para interpretação de Sendín (1999) e os dados normativos de Nascimento (2007). Os resultados obtidos para o agrupamento dos 43 gestores (ver tabela 1) mostram que esses gestores são capazes de simplificar suas percepções e transformá-las em respostas objectivas, sem interferência emocional (Lambda $=.92$ ); e, ainda, que enfrentam os problemas sem se perderem em meticulosidades que poderiam ocasionar comportamentos mais perfeccionistas e com tendência ao detalhe excessi- 
vo, levando à perda de tempo e prejuízos na definição do problema (OBS = $0)$. Significa dizer, a partir do entendimento da teoria do processamento da informação (Hunt, 1980; Newell, Shaw, \& Simon, 1958; Simon \& Newell, 1972), que os processos cognitivos gerais a todas as tarefas cognitivas ou seja, os processos de percepção e aten- ção, estão bastante adequados nesses gestores. Estes processos de percepção e atenção, quando ajustados, permitem a captação, apreensão e evocação da memória de maneira condizente $\mathrm{e}$ apropriada à necessidade, garantindo, em boa parte, a eficiência da codificação que será realizada.

Tabela 1

Resultados nas variáveis do Rorschach quanto ao processamento da informação

\begin{tabular}{crrrrrr}
\hline Variáveis & Média & Desvio Padrão & Mínimo & Máximo & Mediana & Moda \\
\hline R & 23.19 & 10.18 & 14.00 & 52.00 & 19.00 & 14.50 \\
Lambda & .92 & .66 & 0.07 & 4.00 & .75 & .40 \\
Zf & 11.65 & 5.18 & 2.00 & 25.00 & 11.00 & 9.00 \\
Zd & .01 & 4.85 & -13.00 & 8.00 & .50 & ----- \\
W & 8.53 & 4.27 & 1.00 & 18.00 & 8.00 & ----- \\
D & 10.40 & 6.85 & 1.00 & 28.00 & 9.00 & 4.00 \\
Dd & 4.26 & 4.34 & .00 & 17.00 & 3.00 & 1.00 \\
M & 2.56 & 1.82 & .00 & 7.00 & 2.00 & 2.00 \\
DQ+ & 6.12 & 3.66 & 1.00 & 19.00 & 6.00 & 5.00 \\
DQo & 15.81 & 8.91 & 5.00 & 37.00 & 12.00 & 9.00 \\
\hline
\end{tabular}

Ainda, na forma de apreensão da informação, apenas dois dos participantes apresentaram HVI positivo no protocolo do Rorschach, o que não possibilita fazer qualquer afirmação em termos grupais. É possível apenas dizer que estes indivíduos podem utilizar uma forma de percepção dos estímulos e recolha das informações mais cautelosa e selectiva, o que interferirá no estabelecimento de relações com o meio externo e em todo o processamento cognitivo dos dados, derivados desta percepção.

Com o foco na organização da informação recebida, na perspectiva de Mintzberg (1994), outro aspecto obser- vado no Rorschach dos gestores foi a capacidade de apreender os estímulos, utilizando um modo mais organizativo para processar a informação advinda do exterior, aparentando um nível elevado de motivação e iniciativa na resolução do problema $(\mathrm{Zf}=50 \%)$. Associado a isto, a capacidade resolutiva de um indivíduo em relação aos problemas que se lhe apresentam, depende, em termos cognitivos, do grau de destreza nas operações mentais envolvidas, o que se observou na nota $\mathrm{Zd}=.01$ que explica uma recolha e codificação da informação realizada com facilidade e precisão. 
No entanto, constata-se por meio do Rorschach que há nesses gestores uma tendência a observar os estímulos de maneira peculiar, detendo-se em elementos que talvez passem como sem importância para as demais pessoas, parecendo haver uma relação com a busca de exactidão ( $\mathrm{Dd}=18 \%)$, distanciando-se relativamente dos dados óbvios e económicos ( $\mathrm{D}=45 \%$ ), porém, sem perder a visão de conjunto (W = 37\%). Quando observados os dados normativos americanos (Sendín, 1999), poderia se afirmar alguma perda ou distorção na apreensão dos dados. Contudo, se são levados em conta os dados normativos de Nascimento (2007) para a população brasileira de São Paulo, os scores apresentados pelos participantes neste estudo parecem estar absolutamente de acordo com a amostra referida.

Estes dados permitem-nos afirmar que os sujeitos da amostra apreendem de forma cuidadosa a informação. Do mesmo modo, observa-se uma capacidade de organização e a busca por apreender o maior número de informações, sem deixar que nada escape, como características comuns de pessoas com iniciativa e capacidade de resolução face aos problemas do quotidiano $(\mathrm{W}=8.53$ e $\mathrm{M}=2.56$; tipo vivencial extratensivo preponderante na amostra). Esta afirmação pode ser melhor explicada, se for levado em consideração o tempo de experiência como gestor e os conhecimentos que detém na área de actuação da empresa. Segundo Almeida (1994), quando se compara especialistas (experts) e inexperientes (novices), destaca-se que a qualidade dos conhecimentos possuídos, acerca do problema em foco, diferencia o desempenho positivamente, já que o especialista (expert) possui mais informação na memória de longo prazo e a tem melhor organizada e hierarquizada, o que permite acesso mais fácil e rápido para a finalização da resposta a ser emitida (output).

Também é possível dizer que esses gestores apresentam um funcionamento convencional, no que se refere às operações de análise mais complexas de codificação, ranking e evocação da memória de longo prazo para a escolha ou rejeição da resposta (modelo de Exner, 1989; estrutura de processamento da informação resumida em Simon, 1981). As suas respostas ao Rorschach, enquadram-se nos scores da população em geral, sendo que elas expressam um aspecto correcto, sem grande complexidade, mas que reflectem a compreensão do estímulo e capacidade de resolução do problema (DQo $=68 \%)$. Por outro lado, foram emitidas respostas de cunho mais sofisticado e que exigem maior investimento na sua elaboração cognitiva (DQ+ = $27 \%$ ), o que, todavia, não parece garantir brilhantismo ou rendimento superior, como argumenta Silva (2002), mas que, como se vê, retratam a alta produtividade e desempenho desses gestores, ratificando o pensamento de Mintzberg (1994) de que a qualidade da execução do papel informacional define a eficiência do trabalho de gestão. 


\section{CONCLUSÕES}

Os resultados médios obtidos permitem afirmar que os gestores avaliados apresentam, seguindo o Modelo de Processamento da Informação no Rorschach (Exner, 1989), boas condições de recolher dados, de avaliá-los comparando-os às informações armazenadas, estabelecendo rankings para posterior escolha da melhor resposta a ser emitida. E também aparentam ajustada capacidade organizativa, o que favorece o processamento das informações, e condições de serem objectivos e precisos na avaliação dos problemas que se lhes apresentem. Parece mesmo, como sugeriu Mintzberg (1994), que o gestor possui um conjunto de capacidades e conhecimentos que forjam a interpretação do mundo ao seu redor, a partir de um modo de processamento ou um estilo cognitivo próprio e peculiar. É possível que o elevado desempenho esteja relacionado a este estilo pessoal de manejar as informações.

Apesar da tendência à exactidão, não parece que os gestores da amostra se percam em meticulosidades e talvez seja justamente esta a característica que os diferencie e permita o sucesso na execução de suas tarefas, pois não perdem a visão do todo ou a qualidade de suas respostas (output). Como a maioria dos gestores é bastante experiente nas suas funções, parece que, segundo a teoria de Henry Mintzberg, processar informações ininterruptamente e trabalhar na resolução de problemas complexos é uma tarefa essencial do trabalho de gestão, para além de habitual. Desta forma, assim que as manchas de tinta de Rorschach foram apresentadas, os gestores passaram a trabalhar com algo conhecido (um problema complexo a ser resolvido com eficácia) e a executar uma tarefa frequente em seu quotidiano: processar informações e resolver problemas.

\section{REFERÊNCIAS}

Acklin, M. W., \& Wu-Holt, P. (1996). Contributions of cognitive science to the technique: Cognitive and neuropsychological correlates of the response process. Journal of Personality Assessment, 67(1), 169-178.

Almeida, L. S. (1994). Inteligência: Definição e medida. Aveiro: CIDInE.

Chase, W. G., \& Simon, A. H. (1973). Perception in chess. Cognitive Psychology, 4, 55-81.

Exner, J. E. (1974). The Rorschach: A comprehensive system (vol. 1). New York: Wiley.

Exner, J. E. (1978). The Rorschach: A comprehensive system (vol. 2). New York: Wiley.

Exner, J. E. (1989). Searching for projection in the Rorschach. Journal or Personality Assessment, 53(3), 520-536.

Exner, J. E. (1999). Manual de classificação do Rorschach para o sistema compreensivo. São Paulo: Casa do Psicólogo.

Exner, J. E., \& Sendín, C. (1997). Some issues in Rorschach research. European Journal of Psychological Assessment, 13 (3), 155-163.

Gold, J. M. (1987). The role of verbalization in the Rorschach response process: A review. Journal of Personality Assessment, 51 (4), 489-505.

Huitt, W. (2003). The information processing approach to cognition. Educational Psychology Interactive. Valdosta, GA: Valdosta State University. Acedido em Novembro, 23, 2008 a partir de 
http://www.edpsycinteractive.org/topi cs/cogsys/infoproc.html

Hunt, E. B. (1980). Intelligence as an information-processing concept. British Journal of Psychology, 71, 449-474.

Miller, G. A. (1956). The magical number seven, plus or minus two: Some limits on our capacity for processing information. The Psychological Review, 63, 81-97.

Mintzberg, H. (1975). The manager's Job: Folklore and fact. Harvard Business Review, 53(4), 49-61.

Mintzberg, H. (1994). Rounding out the manager's Job. Sloan Management Review, 36(1),11-26.

Mintzberg, H. (2006). MBA? Não, obrigado: uma visão crítica sobre a gestão e o desenvolvimento de gestores. Porto Alegre: Bookman.

Mintzberg, H., \& Gosling, J. (2003). Educando administradores além das fronteiras. Revista de Administração de Empresas, 43(2), 29-43.

Muller, R., \& Turner, R. (2009). Leadership competency profiles of successful project managers. International Journal of Project Management, 28(5). (in press)

Nascimento, R. S. G. F. (2007). Rorschach comprehensive system data for a sample of 409 adult nonpatients from Brazil. Journal of Personality Assessment, 89(1), 35-41.

Newell, A., Shaw, J. C., \& Simon, H. A. (1958). Elements of a theory of human problem solving. Psychology Review, 65, 151-166.

Rapaport, D. (1946). Diagnostic psychological testing. Chicago: Year Book Publishers.

Reitman, J. S. (1976). Skilled perception in GO: Deducting memory structures from inter-response times. Cognitive Psychology, 8, 336-356.

Resnick, L. B. (1976). The nature of intelligence. Hillsdale, NJ: Erlbaum.
Rorchach, H. (1974). Psicodiagnóstico (8 ed.). São Paulo: Editora Mestre Jou.

Schafer, R. (1954). Psychoanalytic interpretation in Rorschach testing. New York: Grune \& Straton.

Sendín, C. (1999). Tríade cognitiva I: Processamento da informação. In J. E. Exner \& C. Sendín (Eds.), Manual de interpretação do Rorschach para o sistema compreensivo (pp. 71-83). São Paulo: Casa do Psicólogo.

Silva, D. R. (2002). O processamento da informação no Rorschach de crianças portuguesas. Psico-USF, 7(1), 35-42.

Silvius, A. J. (2009). Project Management 2027: The future of project management. In T. T. Kidd (Ed.), Handbook of research on technology Project management, planning and Operations, Capt. II, pp.1736, USA.

Simon, H. A. (1981). Informationprocessing models of cognition. Journal of the American Society for Information Science, 364-377.

Simon, H. A., \& Gilmartin, K. (1973). A simulation of memory for chess positions. Cognitive Psychology, 5, 29-46.

Simon, H. A., \& Newell, A. (1972). Human problem solving: The state or the theory in 1970. American Psychologist, 145-159.

Smith, S. R., Bistis, K., Zahka, N. F., \& Blais, M. A. (2007). Perceptualorganizational characteristics of the Rorschach task. The Clinical Neuropsychologist, 21, 789-799.

Sternberg, R. J. (1981). The evolution of theories of intelligence. Intelligence, 5 , 209-230.

Sternberg, R. J. (2000). Psicologia cognitiva. Porto Alegre: Artmed.

Sternberg, R. J. (2003). A broad view of intelligence: The theory of successful 
intelligence. Consulting Psychology Journal Practice and Research, 55(3), 139-154.

Tengblad, S. (2006). Is there a 'New Managerial Work? A comparison with Henry Mintzberg's classic study 30 years later". Journal of Management Studies, 43(7), 1437-1461.

Werlang, B. G., \& Cunha, J. A. (1993). Avaliação da personalidade sob enfoque projetivo. In J. A. Cunha (Ed.), Psicodiagnóstico-R (pp. 123-129). Porto Alegre: Artes Médicas.

Yazigi, L. \& Gazire, P. (2002). Avaliação cognitiva e Rorschach. Psico-USF, 7(1), 109-112. 